\title{
Multi-modal Search Interaction: Helping Users Make Connections Between Conceptual and Spatial Maps
}

\author{
Christian Beck and Craig Birchler \\ School of Informatics 901 East 10th St. \\ Indiana University, Bloomington, IN 47408 \\ \{cmbeck, cbirchle\} @indiana.edu
}

\begin{abstract}
In this paper we describe an interface for searching and conceptualizing related information. We describe a specific application of this interface for searching traditional artists in the state of Indiana. The interface employs multimodal interaction by allowing the user to search for artists using a map of the state of Indiana alongside a conceptual hierarchical map of crafts and subcrafts. The two interfaces work in parallel with each other so that as a user interacts with one interface, it affects what is displayed on the opposing map. The goal of this application is twofold. One goal is to provide users with a better and simpler means for searching a specific set of information using only relevant and related data. The second goal is to provide companies, organizations or institutions with an interface that will allow them to provide clients or targeted users with a more effective way to search their database.
\end{abstract}

Keywords: Conceptual map, spatial map, interactive searching.

\section{Introduction}

We have developed an interface that seeks to help users make more meaningful connections between data while also providing a logical means for conceptualizing data. We have created an interface for businesses or organizations to input data into an application that populates this interface dynamically. Here however, we describe the interaction with the interface. We begin by outlining a small sample of conceptual and spatial networks already in use. We then illustrate the problem we intend on solving with our interface.

\subsection{Examples}

Visual Thesaurus. Plumb's Visual Thesaurus (www.visualthesaurus.com) is a conceptual network that links words based on the connections that link them together. The interface is a joy to use and effectively allows the user to understand more meaningful links between words based on different meanings. However, this application only works for datasets of connected entities based on likeness (in this case, similar words). It is not optimal for conceptualizing other hierarchical or spatial concepts.

Google Maps. Google Earth (earth.google.com) is an interface that allows for easy spatial visualization of basic data. However, while a user may filter data shown on the 
map, it is a one-way relationship. A user can filter data on the map using different parameters but not vice-versa.

Gapminder.org. This site provides a unique and powerful tool for viewing data using multiple attributes. A user can view countries in terms of GDP, time, location and population size. However, whereas we are concerned with conveying a relationship between multiple representations, gapminder.org integrates all representations into a single interface. Additionally, the data is represented for the sake of representation, not for a specific goal such as trying to find a country to visit, for example.

\subsection{Problem}

The examples we have described all succeed more or less in their goals but they only work to a certain extent. We are proposing an interface that can build on examples such as these in order to explore how conceptual maps can be used as searching devices rather than simply for representation and visualization. Our interface provides communication between two conceptual maps in order to allow a user both to conceptualize related data and to create a more meaningful and effective search.

\section{Interface}

Our interface is open to appropriation towards any number of instances that involve the representation of multiple conceptual networks. Here we describe one instance of use for a folklore group that seeks to allow users to search and view their database of traditional folk artists in the state of Indiana. The interface couples a spatial network with a conceptual, hierarchical network. The spatial network highlights counties and cities based on whether they contain folk artists. Each artist will be contained in a county. The county represents the number of artists by becoming a darker color. If a user clicks on a county, the artists will then be laid out according to city. Opposite the map, a conceptual network constructs a hierarchical structure of crafts that are represented by the artists.

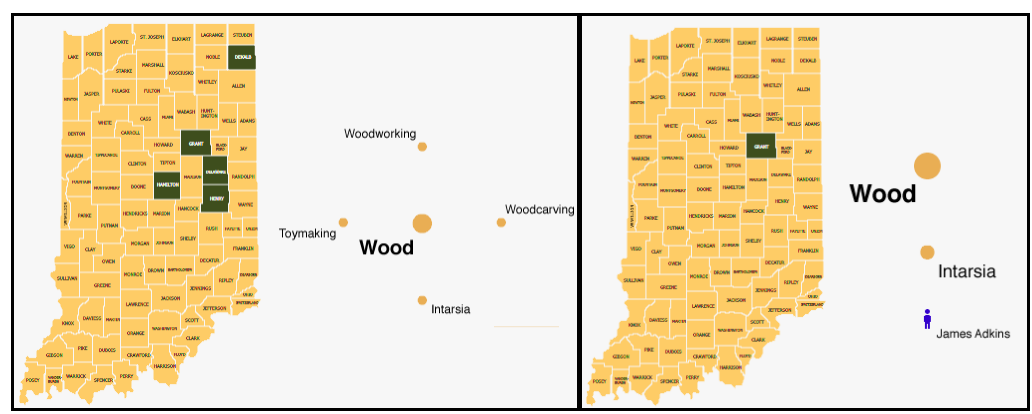

Fig. 1. On the left, a user has refocused on the parent craft 'wood'. Counties with data associated with the network remain highlighted on the map. On the right, a single relevant county remains based upon the user's selection of the sub-craft 'intarsia' from the parent craft of 'wood'. A single data node, 'James Adkins', is present in the network. Data is represented contingent upon the scale of the opposing side. 
The database includes hundreds of sub-crafts that are divided into around twenty parent crafts. For example, a typical craft is glass with a sub-craft of glass-blowing. The system is open to allow for infinite levels but for our particular client's need, in this application we have included two. Once a user clicks on a sub-craft, the craft network is then populated with artists in that area. While a user refines the scales by clicking on counties or crafts, the manipulated network will affect the other as shown below.

The pair of images in fig. 1 shows what happens when a user searches based upon the hierarchical network of crafts. In this instance the user selects the craft 'wood' then the sub-craft 'intarsia'. On the left, this action filters down the counties on the map to show only those that have people associated with the four sub-crafts within 'wood'. In the right image of fig. 1, the available sub-crafts have reduced to 'intarsia' based upon the user's selection. In this case there is only one person shown as a node off of 'intarsia'. Accordingly, only one county is represented. From this, it is clear which county James Adkins resides.

The interface also utilizes the ability for the user to search for craft artists on the map instead of the craft network. In this instance the user could zoom into the map by clicking on a region of Indiana which would then change the parent crafts visible on the network. The highlighted counties would remain unchanged, however the scale would affect the craft network to show only crafts present in that region. Incidentally, the user could select a craft as seen in fig. 1 and the network would then filter counties in that specified region. The interface gives two methods for searching through craft artists while each affects visible data on the opposite side. As a result, the user can better understand the nature of the data, make more meaningful connections between how crafts and geography are related, and search in a way that better represents the nature of the data (e.g. spatially and hierarchically).

\section{Conclusion}

We have described what we feel to be an innovative contribution to HCI-design by presenting a multi-modal, interactive search interface. Existing applications such as Plumb's Visual Thesaurus and Google Earth are innovative and entertaining towards their own goals. However, we have proposed an interface that can build upon the oneway filtering and single data representation. The core component of our interface lies in the power it gives the user to view a set of data in multiple representations. We hope to have accomplished this by creating not only an interaction between the interface and the user but between the different data representations themselves. In doing so we feel this interface provides a more intuitive interaction rather than one that restricts the user by conforming their interpretation to only what the interface provides. For example, our multi-modal interfaces allow users to not only ask questions like, "Where are wood craftspeople in the state of Indiana?" but to be able to follow up with questions such as, "Who practices woodcarving in Hamilton County?" We have attempted to create an interface that will model itself upon how the user conceptualizes data rather than the undesirable alternative of forcing meaning and conception onto the user [1]. 


\subsection{Future Work}

While we believe to have described an innovative contribution to the field of HCI, we have yet to empirically prove our concept. The effectiveness of our application will be determined in two areas: usability studies and user experience. First, usability studies will be developed in tandem with future development of the network visualization. As stated, we are open to development of the application based upon a wide variety of data types, each with their own organizational and user needs. In this instance we have modeled hierarchical and spatial representations. While maps are a commonly understood means of spatial visualization, we do not claim that a wheel of nodes is necessarily best to represent hierarchies. In fact, currently we are creating new methods of representation for the craft network by clustering groups of crafts, and using font-size to convey information density much like tag clouds. Visualization of the data must be contingent upon a combination of the type of data being represented, the basic desires of the organization compiling the data, and the users expected to benefit from it. Multiple variations of data types will need to be categorized so as to aid in development and testing of corresponding visualizations.

Second, we hope to determine effectiveness in terms of the user's ability to develop new connections not readily apparent between types of data visualization. A proof of concept has been derived and, through study of individual development of knowledge based upon the multi-modal interface, we hope to develop supporting data which proves our claims. Knowledge will be measured as a comparison of understanding between using unidirectional and multidirectional interface styles. From these tests we hope to further refine our prototype so that it can be expanded and appropriated for usage in other areas.

Acknowledgments. Thanks to Jeff Bardzell for giving us guidance throughout our development of this application, to Jon Kay for his collaboration on behalf of the Traditional Artists of Indiana (TAI), and Dave Roedl for his coding help.

\section{Reference}

1. Krippendorf, K.: The Semantic Turn. Taylor and Francis Group, London (2006) 